\section{Category}

Chemistry in

Medicine and

Biology

\section{Key words}

multicomponent

reaction

chemical space

automated synthesis

F. SUTANO, S. SHAABANI, C. G. NEOCHORITIS, T. ZARGANES-TZITZIKAS, P. PATIL, E. GHONCHEPOUR, A. DÖMLING* (UNIVERSITY OF GRONINGEN, THE NETHERLANDS) Multicomponent Reaction-Derived Covalent Inhibitor Space Sci. Adv. 2021, 7, DOI: 10.1126/sciadv.abd9307.

\title{
A New Approach for the Synthesis of Covalent Inhibitor Libraries
}

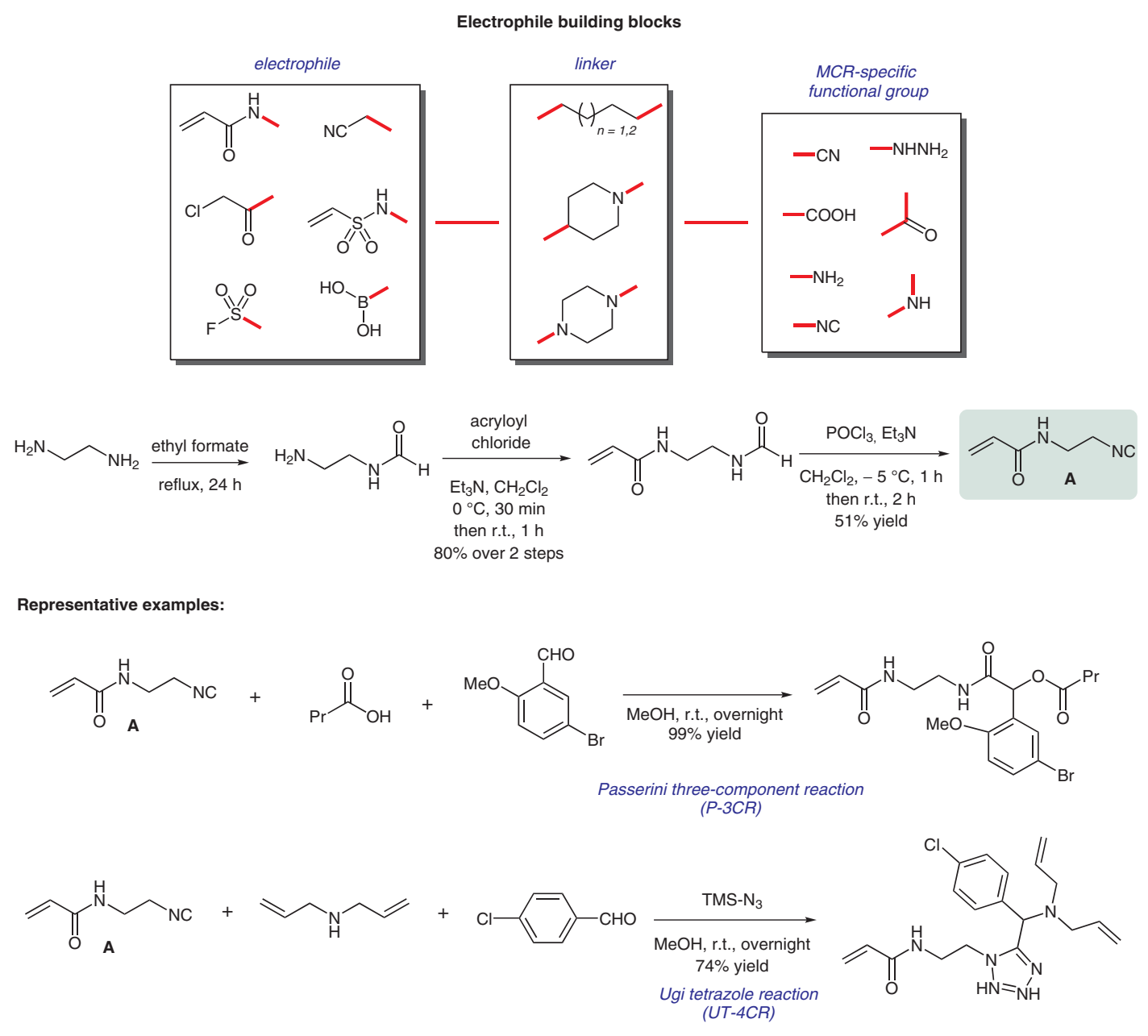

Significance: Ten different multicomponent reactions (MCRs) were used to rapidly access a large library of potential covalent inhibitors. 102 compounds were synthesized by introducing commercially available or readily accessible electrophile building blocks into ten different scaffolds. The synthesis can be performed on millimole scale as well as on nanoscale in an automated fashion with high compatibility to many functional groups. In general, this methodology is simple, mild, and step economical compared with others. This library might find an application in a wide variety of drug development programs.

SYNFACTS Contributors: Dirk Trauner, Andrej Shemet Synfacts 2021,17(05), 0572 Published online: 20.04.2021 DOI: 10.1055/s-0040-1719800; Reg-No.: T02921SF
Comment: The strategy employs bifunctional electrophile building blocks which consist of an electrophile functional group, a linker, and an MCRspecific functional group. The building blocks, if not commercially available, are synthesized on gram scale in one to three steps. For example, the acrylamide isocyanide $\mathbf{A}$ was synthesized in a threestep sequence starting from ethylenediamine. The latter was employed in Passerini three-component and Ugi tetrazole reactions to give the corresponding products in high yield. In addition, bisallylamine and bromo phenyl functionalities could be further used in a ring-closing metathesis or in a SuzukiMiyaura coupling. 